\title{
Family Optics Day: an optics outreach event for the general public
}

\section{Katherine Calabro}

Katherine W. Calabro, "Family Optics Day: an optics outreach event for the general public," Proc. SPIE 8481, Optics Education and Outreach II, 84810Q (18 October 2012); doi: 10.1117/12.930283

SPIE Event: SPIE Optical Engineering + Applications, 2012, San Diego, California, United States 


\title{
Family Optics Day: \\ an optics outreach event for the general public
}

\author{
Katherine W. Calabro \\ Boston University, Student Chapter of OSA/SPIE, 44 Cummington St., Boston, MA, USA 02215
}

\begin{abstract}
As a way to extend the reach of optics education to a larger and more diverse audience, the Boston University student chapter of OSA/SPIE held a 'family optics day'. The outreach event was organized in a manner similar to a science fair, with participates being free to roam between a dozen demonstration stations, each focused on a different area of optics. We highlight the methods of publicity used to attract an attendance close to 300 people, and demonstrate that an event such as this is an excellent way to maximize outreach impact for a given level of organizational effort.
\end{abstract}

Keywords: Optics Outreach, Public Event, Student Chapter, Publicity, Family Event

\section{INTRODUCTION}

Of the numerous educational outreach efforts being pursued specifically for optics, a majority are focused on classroom settings (ex. through teacher training and volunteer visits for classroom demonstrations), or in small organized groups such as Girl Scout troops ${ }^{1,2}$. Unfortunately, this limits exposure to a rather narrow subset of children, and requires significant support from teachers and school administrators. To expand the reach of the traditional classroom model, science fairs such as those held by local communities (ex. Cambridge Science Festival in Cambridge, MA) are attractive alternatives, but are generally very broad with optics being only one limited portion of the entire event ${ }^{3}$. With the support of the New England Section of the Optical Society of America (NES/OSA), the Boston University OSA/SPIE student chapter organized an event, called Family Optics Day, that was devoted entirely to optics, but which was also open to the general public, thus capturing the benefits, and avoiding the limitations, of both approaches.

The concept for Family Optics Day originated from NES/OSA. NES/OSA has participated in numerous science fair type events in the past, where they were generally limited to a single table, being only one of many demonstration stations. To take advantage of their large collection of demonstrations, they proposed an event specifically for optics which would also allow for more involved explanation of each of the demos ${ }^{4}$. The original intended audience was professional members of the NES/OSA and their families. As the common member on both the NES/OSA council and BU student chapter, I suggested a joint effort as a way to recruit more (student) volunteers, and to expand the event audience to families of BU students, faculty, and staff. As planning progressed, the scope of the audience soon grew to include the general public. With this structure in place, the goals of the event were then defined by the following: 1) expose youth K-12 to optics through demonstrations, 2) involve parent participation, 3) attract a diverse audience, and 4) increase awareness of both NES/OSA and the BU OSA/SPIE student chapter.

This manuscript details the successful organization and carry through of Family Optics Day, including event specifics, demonstrations, budget, and of particular interest, publicity, which brought in close to 300 people.

\section{EVENT DETAILS}

Family Optics Day was held on Saturday September $17^{\text {th }} 2011$ from 11 AM to 2 PM in a large meeting space in the Photonics Center at Boston University. Ten demonstration stations were set up around the room with at least one NES/OSA or BU student volunteer stationed at each. We had a total of 18 volunteers. Attendees were invited to roam among the tables where the volunteers would either show them a demonstration, or help them build a take-home project. A snack table was also available offering a free assortment of finger foods (Figure 1).

Optics Education and Outreach II, edited by G. Groot Gregory, Proc. of SPIE Vol. 8481

84810Q · (c) 2012 SPIE · CCC code: 0277-786/12/\$18 · doi: 10.1117/12.930283 


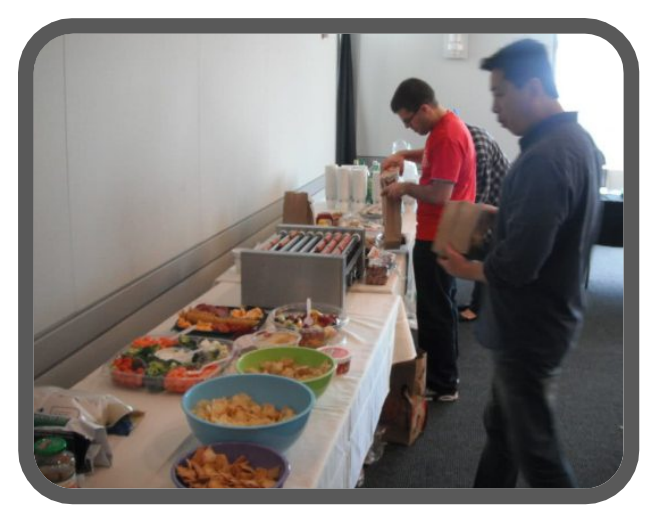

Figure 1 Snack Table

\section{DEMONSTRATIONS}

As previously mentioned, NES/OSA already possessed a large number of optics demonstrations that are used during other outreach events. Many of the demos are similar to those found in the popular 'optics suitcases,' such as those available through the Institute of Optics at the University of Rochester, and the Optics Institute of Southern California. Volunteers from the student chapter at MIT, BAE systems, and the Northeastern University RE-SEED (Retirees Enhancing Science Education through Experiments and Demonstrations), also donated their time and talents by bringing several other demonstrations. The ten demonstration tables can be classified into three categories: take-home projects, hands-on activities, and observational demonstrations. Each is described here:

- Take-Home Projects:

○ UV Fluorescent Beaded Bracelets: The concept of fluorescence was explained using beads excited by UV light, which participants could make into a bracelet to keep. Beads were purchased through teachersource.com

○ Kaleidoscopes: To complement NES/OSA's popular 'Giant Kaleidoscope' (Figure 2), each child was given the materials to build their own smaller version using kits purchased from discountschoolsupply.com.

- Hands-On Activities:

- Colors: The concepts of both additive and subtractive colors were demonstrated using glow sticks (combine green red and blue to create white light), and colored water (combine cyan magenta and yellow to create a color close to black), respectively.

○ Jello-Optics (ray-optics): Laser pointers and Jello pieces cut into various shapes were used to illustrate the concepts of Snell's law, lenses, and fiber light guides.

- Polarization: Sheets of linear polarizers and birefringent materials (like tape and extruded plastics) were used to demonstrate the polarized nature of light, and the birefringent properties of certain materials.

- Light Maze: The SPIE student chapter of MIT brought a light maze (Figure 2) in which participants were encouraged to position mirrors to direct a light beam through the maze.

o Diffraction: Small diffraction gratings were used to observe the spectrum of colors from natural light in comparison with the spectrum from fluorescent bulbs, demonstrating how white light can be decomposed into different colors.

- Observational Demonstrations:

- Bio-Optics: The use of optics for biomedical applications was demonstrated with a pulse-oximeter, and with an optical measurement system used for clinical research at BU. The effects of light scattering were specifically described.

- IR Imaging: A representative from BAE systems provided a demonstration concerning infrared light, and how it can be used to image non-visible information such as heat.

○ Wireless communications: A demo called 'Light on a sound beam' illustrated how sound information can be transformed into a light signal, which without wires, can be sent through the air to a detector, and then transformed back into sound.

Volunteers at each demo station were trained in advance to explain the material in different ways depending on the age of the child. 

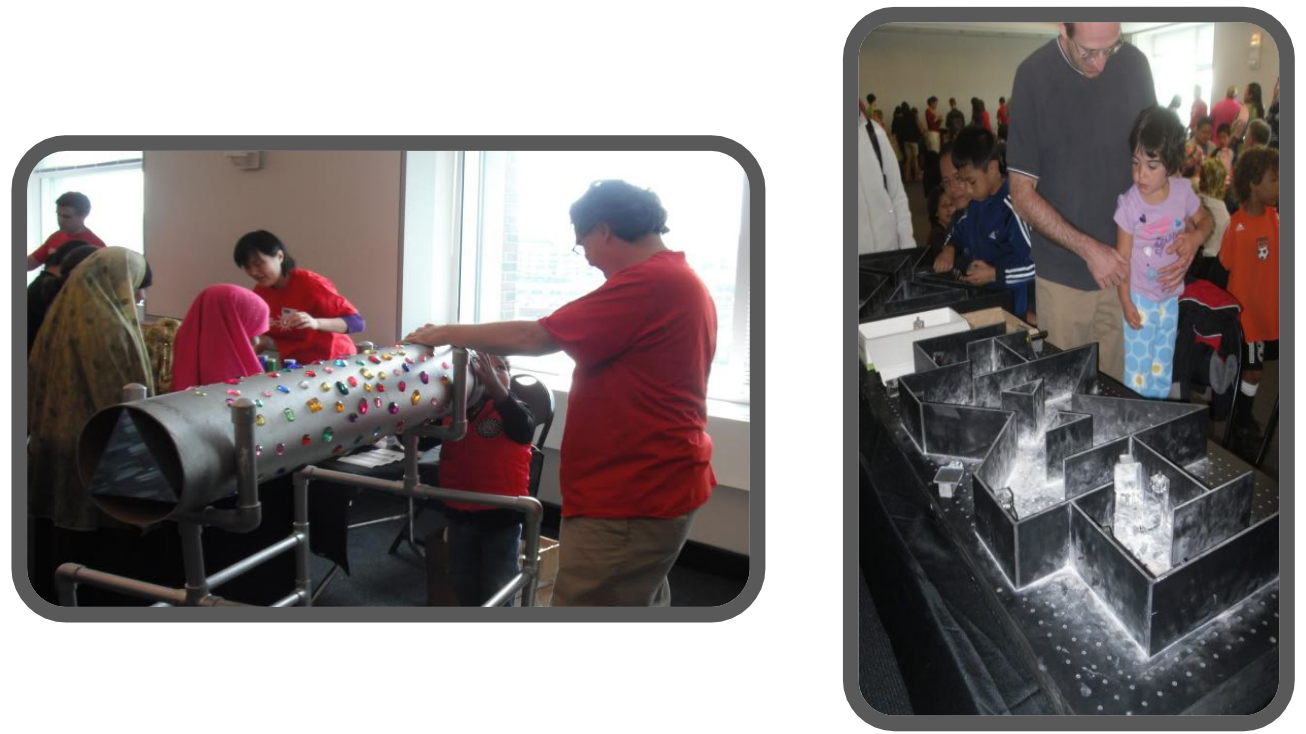

Figure 2: Giant Kaleidoscope (left), Laser Maze (right)

\section{PUBLICITY}

The primary challenge of Family Optics Day was publicity. E-mail messages were used extensively, going out to the NES/OSA membership, and the Boston University science and engineering communities. However, we believe the majority of attendees heard about the event through alternative means such as word of mouth, e-mail advertisements that we distributed to the science departments of local Boston area schools, and online event posting forums.

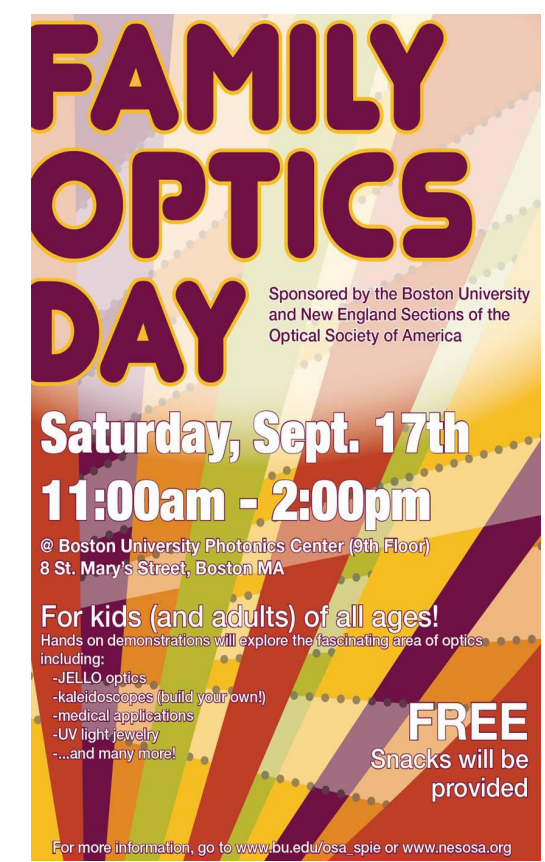

Figure 3: Family Optics Day Advertizing Poster

Several of the online event posting sites were focused primarily for parents looking for local family events (bostoncentral.com and boston.parenthood.com), which gave us direct access to our target audience, and were free to post on. We also posted on a third, more general events page, whofish.com, on which we saw a significant amount of interest as measured by the number of times someone would forward the link to a friend. Some of the other 
organizations that we contacted via e-mail were local parenting groups, including a home schooling network. All of our advertizing communications included a flyer for our event which was created for our group by a friend who is a graphic designer (Figure 3). The result of these efforts was a diverse audience of close to 300 people. Because nearly all of our efforts were made online, our advertizing expenses were very low, and could be completed very efficiently (in terms of time).

\section{BUDGET}

The funding for this event was provided through an Optical Society of America student chapter outreach grant. A breakdown of our expenses is provided in Table 1. With close to 300 people in attendance, this budget breaks down to approximately $\$ 3$ per person. With a smaller budget, costs could easily be reduced by limiting food and volunteer tshirts, without taking away from any of the demonstrations, making this kind of event even more affordable.

Table 1: Event Budget

\begin{tabular}{|c|c|}
\hline Budget Item & Cost \\
\hline Food & $\$ 380$ \\
\hline Take-Home Projects & $\$ 160$ \\
\hline T-shirts (for volunteers) & $\$ 150$ \\
\hline Miscellaneous & $\$ 105$ \\
\hline Demonstrations & \$65 + NES/OSA Optics Suitcase \\
\hline Publicity (whofish.com) & \$35 \\
\hline Facilities & Donated by BU \\
\hline TOTAL & $\mathbf{\$ 8 5}$ \\
\hline
\end{tabular}

\section{CONCLUSIONS}

Particularly for a first time event, Family Optics Day was a successful optics outreach event that exposed a large and diverse audience to optics, with a very reasonable amount of effort. This model could be easily implemented by other groups, particularly if they already have some optics demonstrations. It is particularly well suited for student groups since organizing events at off campus locations can be logistically complicated, especially regarding transportation. It also serves as an advertizing mechanism for the group itself; awareness of our student chapter increased significantly afterwards, and we were thanked personally by the BU Photonics Center director for generating good publicity for the center.

Of course the true benefits- exciting kids about optics- are difficult to identify or quantify. But from informal discussions with attendees, both parents and children seemed very excited to be participating, many asking if it would be a recurring event. A teenage girl who had previously been apathetic about science even informed her mom after the event that she had changed her mind- that 'optics was pretty cool, and everyone would like it a lot more if they taught it like this in school.'

In the future, several improvements could be made to make the event even more successful. Our largest challenge on the day of was a much larger attendance than was expected (expected: 100 people); because of this we ran out of food. It would be helpful to have a general idea of attendance ahead of time, which could be accomplished with an anonymous online interest survey. Additionally, the age distribution of children was much lower than expected, and so in the future it would be beneficial to tailor the demonstrations and their explanations to a younger crowd.

\section{REFERENCES}

[1] Donnelly, J., Hanes, F., Massa, N., "Professional development in photonics: the advanced technology education projects of the New England Board of Education", Proc. SPIE 6668, (2007).

[2] http://www.girlscouts.org/mixitup/PDFs/lighten_up.pdf (26 August 2012)

[3] http://cambridgesciencefestival.org/Home.aspx (26 August 2012)

[4] Gregory, G., Biss, D.P., Darnell, B., “Optical demonstrations through science fairs”. Proc. SPIE 7783, (2010). 